\title{
Regulation of ROP GTPase Signalling at the Gene Expression Level: A Review
}

\author{
Attila Fehér*, Manuela E. Jurca, Csilla Fodor-Dunai and Dulguun Dorjgotov
}

Institute of Plant Biology, Biological Research Center, H.A.S., Szeged, Hungary

\begin{abstract}
Research of plant RHO-type (ROP) GTPases has considerably accelerated during the last few years. Now it is clear that these small proteins play central roles as signalling molecules during many basic cellular processes including cell shape determination, polar growth and responses to hormones, stress factors or pathogens. ROP GTPases have the potential to interact with a plethora of regulators and effectors that finally determines their signalling specificity. These proteins, similarly to the ROP GTPases themselves, are coded by small gene families increasing the complexity of the regulation. The comparison of gene expression patterns of the individual members of these gene families may help to reveal potential signalling chains with biological relevance. In this review previous observation on the differential expression of ROP GTPase genes in various organs and during various developmental processes is summarized. In addition, an inventory of presently known ROP GTPases-interactor proteins is provided with brief descriptions and with correlative comparison of gene expression patterns based on available microarray data.
\end{abstract}

Keywords: Expression pattern, RHO GTPase, ROP regulator, ROP effector, microarray data, protein interaction.

\section{INTRODUCTION}

Small (20-40 kD) RAS-family GTP-binding proteins are key components of various regulatory networks in eukaryotic cells [1]. These proteins, with intrinsic GTPase activity, cycle between GTP- and GDP-bound forms and their nucleotide-bound state strictly determines their cellular activities. The structurally very similar RAS-type GTPases can be divided into several subclasses (RAS, RAN, RAB, ARF, RHO) based primarily on their structure and cellular function [2]. The family of RHO-type GTPases is known to be involved especially in the regulation of the cytoskeleton, reactive oxygen generation and gene expression [3, 4].

RHO GTPases are well conserved among all eukaryotes considering their overall structure and cellular roles as well [5]. However, due to the early split of viridiplantae from the animal-fungal-amoebozoa lineage, RHO ("RHO-of-plant" or ROP) GTPases of plants occupy an unique position in the family. In addition to their somewhat divergent primary structure, the upstream as well as downstream signalling steps, associated with ROP GTPase activity, also have plantspecific features $[5,6]$.

Investigations of ROP GTPases have attained considerable interest during the last years. ROP function was found to be associated with key cellular events in plants including the establishment of cell polarity, polar growth and cell expansion, vesicular trafficking, cell wall synthesis, hormonal signalling, and production of reactive oxygen species in response to pathogen attack (for recent reviews see [7, 8]).

The increasing number of ROP-interacting upstream regulatory and downstream effector proteins (see Fig. 1 for the scheme of ROP-mediated signalling) delineates complex

*Address correspondence to this author at the Biological Research Center, Temesvári krt. 62, 6726 Szeged, Hungary; E-mail: fehera@brc.hu regulatory networks involved in the above processes [6]. Furthermore, the cycling of RHO family proteins between the inactive GDP- and active GTP-bound forms is not only dependent on protein-protein interactions [9] but is also coupled to intracellular translocation events $[10,11]$. Therefore the post-translational regulation of the activity of ROP GTPases is of primary significance concerning their signal transduction potential.

However, as all components of ROP-mediated signalling pathways are coded by small gene families, the importance of transcriptional regulation can not be ruled out, especially in establishing the specificity of signalling components in certain developmental or biological processes [12].

Here we evaluate and correlate available gene expression data to highlight the diversity as well as the specificity of ROP GTPase signalling networks in plants.

\section{PROTEINS INVOLVED IN ROP-MEDIATED SIG- NALLING AND THEIR EXPRESSION}

\section{Differential Expression of ROP GTPases}

Arabidopsis thaliana (L) Heynh. has a family of 11 ROP GTPases, all of them with a very similar overall structure (for reviews [13, 14]; for the used nomenclature see [14]). It is not surprising therefore that the regulation of specific developmental events by specific isoforms requires cell type or tissue-specific expression of the corresponding ROP genes. For example, AtROP1 is expressed only in pollen and flowers [15], whereas AtROP7 is expressed exclusively in roots, stems and hypocotyls [15] and its expression is downregulated following ethylene treatment [16]. Recently, the promoter of AtROP7 was analyzed in transgenic Arabidopsis plants and was found to limit reporter gene expression to the xylem during the late stages of xylem differentiation [17]. AtROP7 was also implicated in secondary xylem formation by an in silico gene expression analysis approach [18]. At- 


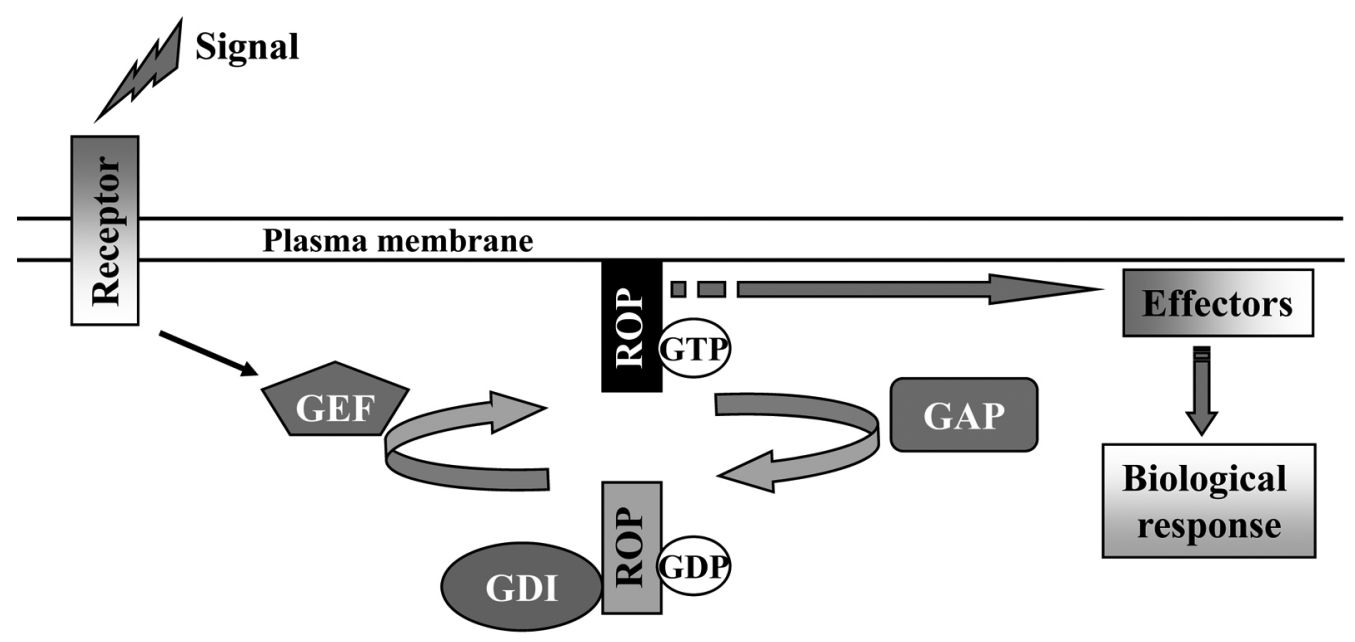

Fig. (1). Schematic drawing of the ROP GTPase cycle and related signalling steps. Various signals may activate receptors, most likely receptor-like kinases, that initiate ROP GTPase-mediated signalling through the activation of ROP GEFs. ROP GEFs facilitate GDP-to-GTP exchange and therefore activate ROPs. Active and membrane- associated ROPs mediate the signal towards various effectors (see text for details) which further transfer the signal for cellular targets evoking the biological response. GAPs and GDIs take part in the inactivation of ROPs by accelerating GTP hydrolysis and by stabilizing the ROP in GDP-bound state as well as sequestering the protein from the membrane, respectively.

ROP4 is ubiquitously expressed in all Arabidopsis organs $[15,19]$ while AtROP6 is absent from closed flowers and siliques but it is expressed in open flowers and in vegetative tissues [19]. Moreover, it is present at a much higher level in guard cells than in the surrounding epithelial cells [20].

There are many indications on developmental stagespecific gene expression of ROP GTPases in various other plant species in addition to Arabidopsis. In Lotus japonicus $L$., one of the two ROP GTPases expressed in root nodules exhibited a strong increase in its transcript level during root nodule development [21]. Three Medicago sativa L. ROP GTPase genes are, although active throughout the plant, differentially expressed during somatic embryogenesis and in response to Rhizobial infection of roots [22]. One of the members of the cotton (Gossypium hirsutum L.) ROP family, GsRAC13, is specifically expressed in cotton fibres undergoing transition from primary to secondary cell wall synthesis, while another one, GhRAC1, is specifically enhanced in the elongation phase of fibre development and declines thereafter [23]. In addition, this gene is abundantly expressed in other rapidly growing cotton tissues. The Zinnia elegans Jacq. ZeRAC2 mRNA molecules were demonstrated to accumulate preferentially in xylem parenchyma and tracheal element precursor cells [24].

In Brassica napus L., the expression pattern of five ROP GTPase genes has been investigated in various organs as well as during androgenesis [25]. In addition to the pollenspecific expression of BnROP5, the contrasting expression level of BnROP5 and BnROP9 during embryogenic cell formation from microspores could be established.

In grape (Vitis vinifera L.), differential expression of the seven investigated ROP genes during grape development as well as in response to various growth regulators in suspension cultured cells has been established [26].

As monocots are considered, a survey of ROP-mRNA abundance in maize, based on multiplex reverse tran- scriptase-polymerase chain reaction and a massively parallel signature sequencing database, showed significant spatial and temporal overlap of the nine (maize has only nine ROP genes) transcripts, with high levels of all mRNA in tissues in which cells are actively dividing and expanding [27]. However, only a subset of maize ROPs is highly expressed in mature leaves and pollen [27]. Rice ROP genes have also been shown to be regulated during anther development and in various organs [28]. OsRACB gene expression has been shown to be strongly up-regulated by salt stress in roots, but not in shoots and leaves [29]. Promoter analysis verified salt inducibility and revealed that the promoter can also be activated by salicylic acid but not by abscisic acid [29].

The above examples clearly indicate that the signalling specificity of a given ROP GTPase is strongly dependent on its cell- and tissue-specific expression.

However, not only ROP GTPases but their numerous interacting protein partners can be transcriptionally regulated increasing the signalling specificity of ROP GTPase-related networks. Although information on ROP regulators and effectors continuously increases, the data on their gene expression pattern are still limited. Below we provide an up to date inventory of ROP regulators and effectors with a short functional description and the available protein-protein interaction as well as gene expression data. The detailed functional characterization of these proteins has already been described in several excellent reviews recently [5-8, 30].

\section{The Regulators of ROP GTPase Activity}

The GTPase cycle of RHO GTPases is facilitated by three types of proteins: the nucleotide exchange factors (GEFs), the GTPase activating proteins (GAPs) and the guanine nucleotide dissociation inhibitors (GDIs) (for a review see [9]). These regulatory proteins are also the products of small gene families in Arabidopsis (for reviews [5, 6], Table 1). 
Table 1. ROP regulators and their experimentally indicated interactions with ROPs (only Arabidopsis genes are given, even if the interaction of homologous proteins was detected in other species; Nt Nicotiana tabacum, At Arabidopsis thaliana)

\begin{tabular}{|c|c|c|c|c|c|}
\hline Annotation & Name & AGI & Affy ID & Interaction & References \\
\hline \multirow{4}{*}{ 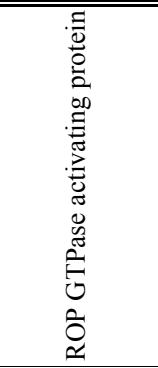 } & GAP1 & At4g03100 & 255410_at & AtROP1 & [31] \\
\hline & GAP3 & At2g46710 & 266324_at & AtROP1 & [31] \\
\hline & GAP4 & At3g11490 & 259287_at & AtROP2 & {$[32]$} \\
\hline & GAP6 & At2g27440 & 265666_at & & \\
\hline \multirow{2}{*}{ 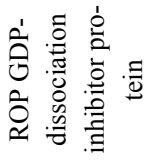 } & GDI1 & At3g07880 & 258637_at & AtROP4 & [33] \\
\hline & GDI2a & At1g62450 & 265115_at & NtRAC5 & [34] \\
\hline \multirow{8}{*}{ 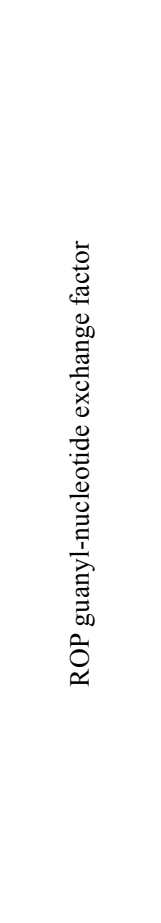 } & GEF3 & At4g00460 & 255757_at & & \\
\hline & GEF4 & At2g45890 & 266913_at & & \\
\hline & GEF5 & At5g05940 & 250756_at & & \\
\hline & GEF6 & At3g55660 & 251778_at & & \\
\hline & GEF7 & At5g02010 & 251080_at & & \\
\hline & GEF8 & At3g24620 & 258129_at & AtROP4 & {$[37]$} \\
\hline & GEF9 & At4g13240 & 254757_at & AtROP1 & {$[36]$} \\
\hline & SPIKE1 & At4g16340 & 245492_at & $\begin{array}{c}\text { AtROP2, } 3,4,5,8,10 \\
11\end{array}$ & {$[38,39]$} \\
\hline
\end{tabular}

In the Arabidopsis genome three genes encoding ROP GDI-like proteins can be found [6]. The approximately 22 $\mathrm{kDa}$ proteins contain highly conserved amino acids in their isoprene binding pocket and exhibits $29 \%$ to $37 \%$ similarity to known mammalian homologues [40]. Most RHO GTPases are post-translationally modified by isoprenylation of the cysteine residue within a C-terminal CAAX box [11]. This lipid modification is responsible for the association of RHO GTPases with appropriate membrane regions where they exert their function [41]. RHOGDI proteins interact with and are able to remove prenylated RHO GTPases from membranes and form cytoplasmic heterodimers with them [41]. In this way, they negatively regulate the signalling activity of RHO GTPases.

Using the yeast two-hybrid system and in vitro assays, it could be demonstrated that AtRHOGDI1 specifically interacts with both AtROP4 and AtROP6 [40]. AtRHOGDI1 ex- pression was found to be ubiquitous in the Arabidopsis organs [40]. Co-expression of the AtRHOGDI1 gene with the NtRACl gene in transfected tobacco protoplasts repressed NtRAC1-dependent auxin-responsive promoter activation, indicating that these proteins indeed function as negative regulators of ROP GTPase signalling in planta [42]. Altered ("more cytoplasmic") intracellular localization of the GTPase if co-expressed with the GDI gene was also observed [42].

AtRHOGDI1 was also implicated in the spatial regulation of root hair growth and was hypothesized to control the activity of the NADPH-oxidase (RHD2/AtrbohC) complex in this context [33].

Nicotiana tabacum L. Nt-RHOGDI2 has been shown to be highly and specifically expressed in elongating pollen tubes where it regulates the polar localization of the Nt- 
RAC5 GTPase and pollen tube expansion [34]. Although NtRHOGDI1 has also been shown to interact with Nt-RAC5, it is not expressed in flower or pollen where Nt-RAC5 is ex- pressed at the highest level, but both proteins are expressed in the shoot apex and young leaves where they may have functional interaction [43].

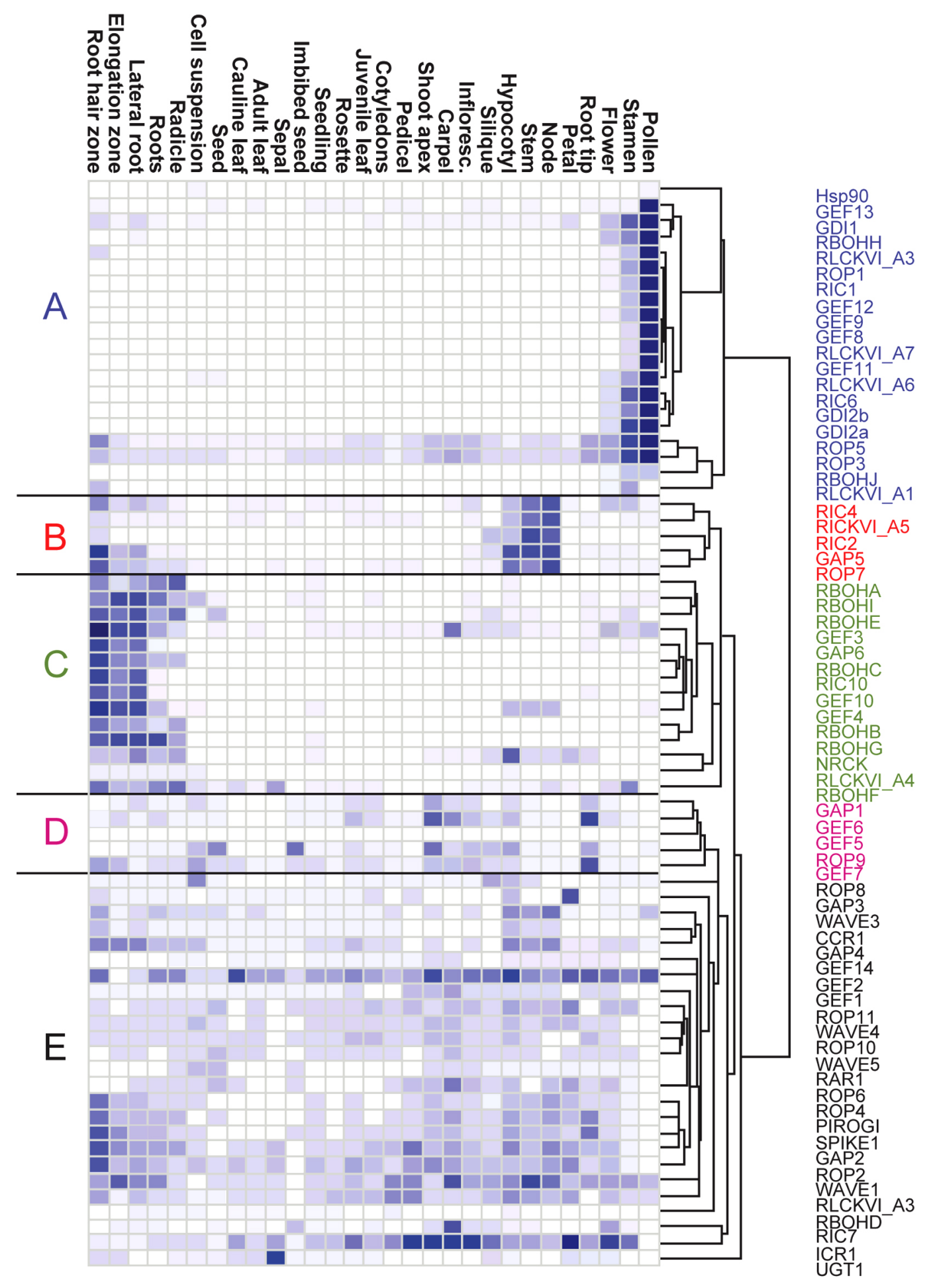

Fig. (2). Expression pattern of ROPs and their known/hypothesized regulators and effectors. Relative gene expression is shown based on microarray data stored in the Genevestigator $[75,76]$ database and analyzed by a hierarchical clustering algorithm of the Genvestigator tool. Darker blue color means higher relative expression. Main clusters (A-E) are separated by lines and the corresponding genes are highlighted by the same color. 
The Arabidopsis genome encodes six GAP proteins. GAP proteins accelerate the hydrolysis of RHO-bound GTP and therefore promote the inactivation of RHO GTPases [44]. All Arabidopsis GAPs contain the conserved CRIB (CDC42/RAC-interactive binding) motif playing a role in binding the GTPase [31].

Point and deletion mutants in the CRIB region reduced the intrinsic GAP activity in vitro, documenting a critical role of this protein interaction motif in plant GAP activity [31]. In tobacco pollen tubes, Nt-RHOGAP1 has been shown to be localized subapically and hypothesized to restrict NtRAC5 activity to the apical region [45] in concert with NtRHOGDI2 [46]. A negative feedback loop including AtROPGAP4 and AtROP2 was revealed in Arabidopsis seedlings subjected to oxygen deprivation. It was shown that ROPGAP4 transcription is increased due to the active AtROP2-dependent production of $\mathrm{H}_{2} \mathrm{O}_{2}$ [32].

Guanine nucleotide exchange factors (GEFs) catalyze the dissociation of GDP from the inactive RHO proteins and allow the binding of GTP, which induces a conformational change that permits interaction with downstream effectors $[9,12]$. Due to this activity, GEFs are primarily responsible for linking cell-surface receptors to RHO protein activation in metazoa [12]. Plants do not contain homologues of DH (Dbl-homology) and $\mathrm{PH}$ (pleckstrin homology) domaincontaining GEF proteins characteristic for animal cells. However, recently, a specific family of plant proteins, carrying the PRONE- (plant-specific ROP nucleotide exchanger) domain exhibiting GEF-activity, has been revealed $[35,36$, 47]. Five out of the 14 PRONE-containing Arabidopsis GEFs are specifically expressed or enriched in pollens [48]. These proteins have a specific conserved C-terminus implicated in the regulation of their activity [48]. Among the other ROPGEF genes there are two which are root specific while the others are expressed in vegetative organs as determined by RT-PCR analysis [49] in agreement with microarray data (see Fig. 2).

A distinct protein with ROPGEF activity was also identified as SPIKE1 in Arabidopsis $[39,49]$. This protein, homologous to the human DOCK family RHOGEFs [50], was shown to control actin-dependent morphogenesis through the heteromeric WAVE and ARP2/3 complexes [39, 49].

\section{Effectors Downstream of ROP GTPases}

Exchange of the hydrolyzed GDP for GTP results in a conformational change of GTPases, unmasking structural domains by which they can bind to their target proteins, the effectors. Many effectors of yeast and animal RHO-type GTPases are well known [51]. RHO and CDC42/RAC GTPases bind different effectors and are involved in specific but interlinked signalling cascades in animal cells. In plants, a single group of RHO-type GTPases, the ROP proteins, has to fulfil all of RHO functions, and it can even be hypothesized that certain roles of the missing mitogen-activated RAS proteins are also played by ROPs in plants [30].

In spite of the high level of structural similarity of plant ROP GTPases to mammalian CDC42/RAC proteins, there are considerable differences as their possible effectors are concerned [51]. One of the most characteristic differences is that in plants no protein kinases carrying the CRIB-motif and directly activated by ROP GTPases could be identified. In animal cells, mitogenic signals are mediated by RAS that activate the RAF-MEKK-MAPK protein kinase cascade, while CDC42 and RAC activate another specific class of protein kinases, the members of the p21-activated kinase (PAK) family, containing the CDC42/RAC interactive binding (CRIB) motif [62, 63]. While the RAS-RAF-MEKKMAPK pathway may be specific to metazoa, PAK kinases regulated by $\mathrm{CDC} 42$ are present also in yeast; with MAP kinase cascades among their effectors [64].

The lack of RAS from plants as well as the significance of animal and yeast CDC42/RAC proteins in protein kinase signalling strongly support the hypothesis that ROPs are also linked to protein kinase cascades in plants. In rice, OsRAC1 was shown to be required for OsMAPK6 protein accumulation and activity in response to elicitor treatment, indicating the role of OsRAC1, and potentially other ROPs, in the activation of MAP-kinase cascades [65]. In the same study it was also proved that the constitutively active OsRAC1 protein occurs in the same protein complex together with OsMAPK6.

Recently, the direct interaction of plant ROP GTPases with receptor-like cytoplasmic kinases (RLCK class VI) has been reported in Arabidopsis [59], and could also be detected in alfalfa (Dorjgotov D and Fehér A, unpublished). This kinase family has 13 members belonging to two groups in Arabidospsis [66]. The Arabidopsis RLCK-VI genes are rather ubiquitously expressed in plant development, but some members are strongly expressed in the pollen $[59,66]$. In addition, the expression of one of the ROP-interacting RLCK VI kinases was shown to be predominantly associated with vasculature and upregulated in leaves exposed to pathogens [59].

A further kinase designated as cysteine-rich receptor kinase (NCRK) belonging to a distinct kinase family has also been shown to interact with ROPs [59]. None of these plantspecific ROP-interacting kinases has any characteristic domain or motif that could be correlated with their ability to bind ROP GTPases.

AtROP5 (AtRAC2) was found to physically associate with a phosphatidylinositol monophosphate kinase (PtdIns PK) activity [67]. This observation indicates that lipid kinases can also be potential ROP effectors in plants similarly as in animal cells [68], although the direct interaction of this type of kinases with ROPs needs to be experimentally verified.

Plants have a family of small CRIB-containing proteins called RICs (RHO-interacting CRIB-motif containing proteins) [53]. RICs are represented by eleven members in Arabidopsis and are highly divergent in sequence outside of the CRIB region. Overexpression of various RIC proteins in pollen tubes causes distinct phenotypes indicating specific functions for the individual RICs [53]. That RICs are indeed downstream targets of ROP GTPases could be the best demonstrated during the establishment of epidermal cell shape in Arabidopsis by the concerted action of AtROP2/4, AtRIC1 and 4 [52]. RIC proteins have been implicated in actin polymerization, microtubule bundling, $\mathrm{Ca}^{2+}$ fluxes during polar growth, cell expansion and morphogenesis $[54,69,70]$. The majority of RIC genes (RIC1,2,4,7,9) is constitutively expressed in various parts of Arabidopsis plants; only RIC3, RIC5, and RIC6 are flower specific [54]. The RIC10 
Table 2. ROP effectors and their experimentally indicated interactions with ROPs. ROPs (only Arabidopsis genes are given, even if the interaction of homologous proteins was detected in other species; Os Oryza sativa. At Arabidopsis thaliana)

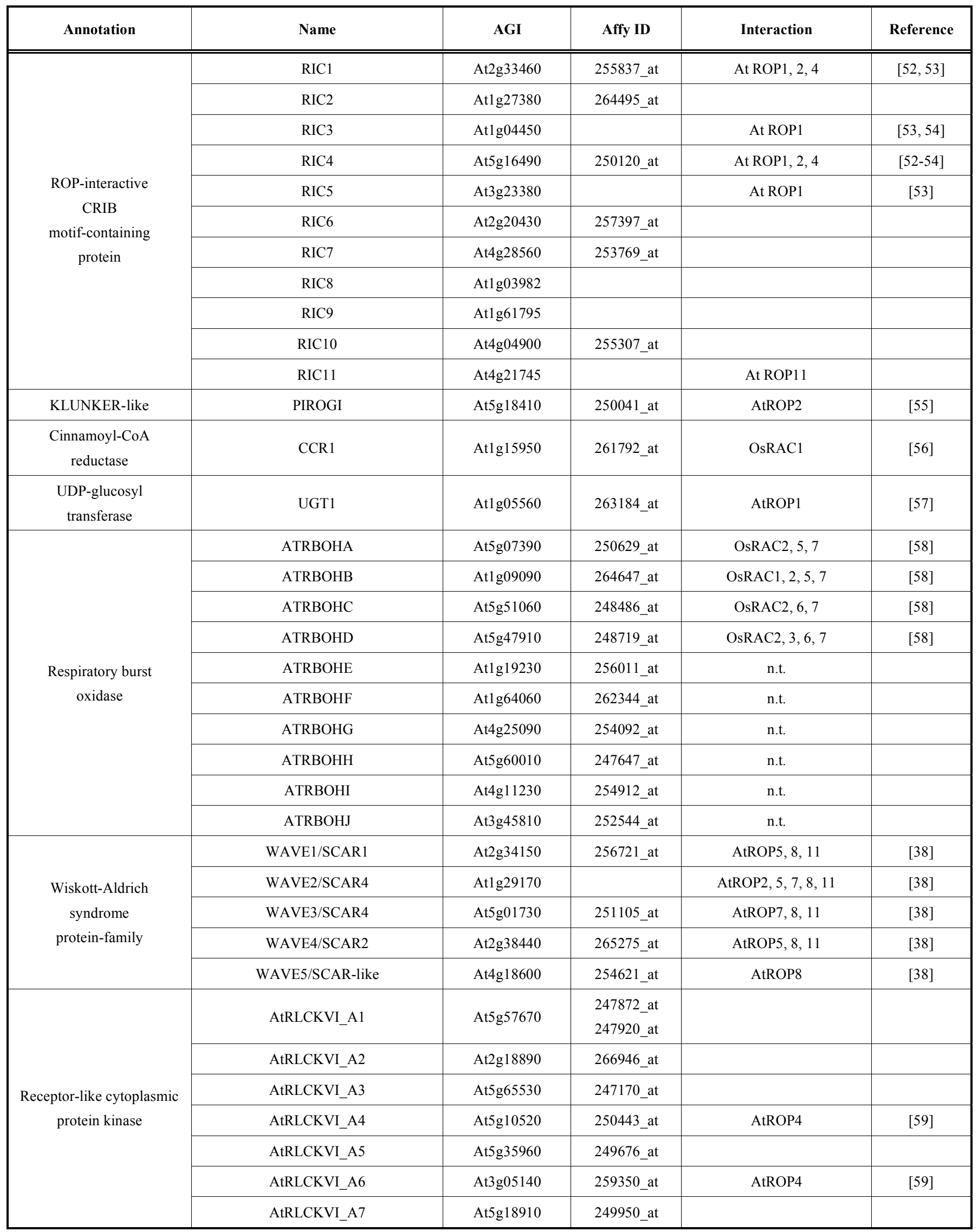


(Table 2). Contd.....

\begin{tabular}{|c|c|c|c|c|}
\hline Annotation & Name & AGI & Affy ID & Interaction \\
\hline \hline $\begin{array}{c}\text { Novel cystein-rich } \\
\text { protein kinase }\end{array}$ & NRCK & At2g28250 & 265545_at & AtROP4, 11 \\
\hline $\begin{array}{c}\text { Tropomyosin-related } \\
\text { protein }\end{array}$ & ICR1 & At1g17140 & 262538_at & AtROP6, 10 \\
\hline $\begin{array}{c}\text { Required for Mla12 } \\
\text { resistance }\end{array}$ & RAR1 & AT5G51700 & 248379_at & OsRAC1 \\
\hline Heat shock protein 90 & Hsp90 & At5g52640 & 248332_at & OsRAC1 \\
\hline
\end{tabular}

transcript appeared to be more abundant in leaves and roots but was expressed weakly in flowers, inflorescences, stems, and siliques [53].

In the absence of any recognizable enzyme activity of these very small (116-224 amino acids) proteins, it is very likely that they serve as adaptors to link ROP GTPases to effectors [6]. The nature of these RIC-dependent effectors still remains to be elucidated.

In addition to RICs, there are further plant-specific downstream targets of ROP GTPases. A recently discovered interacting partner of the active AtROP1 GTPase, ICR1 (interactor of constitutive active ROPs 1 ; [60]), may also serve as an adaptor protein linking ROP GTPase signalling to the exocyst complex involved in exocytosis-related vesicle trafficking and membrane fusions.

The Arabidopsis UDP-glucose transferase (UGT1) directly binds AtROP1 and via this interaction ROP1 may indirectly regulate callose synthase activity and the formation of the new cell wall during cytokinesis as UGT1 transfers UDP-glucose to the catalytic subunit of callose synthase [57]. Another plant-specific ROP effector, cinnamoyl-CoA reductase, has also a role in cell wall formation, namely, it catalyses the NADPH-dependent synthesis of lignin monomers [57].

In rice (Oryza sativa L.), the OsRAC1 GTPase has been found to form complex(es) with the RAR1 (required for Mla12 resistance) protein as well as the heat-shock proteins, HSP90 and HSP70, during the pathogen-triggered innate immunity response of the plant [61].

There are only few known RHO effectors that are moreor less conserved between plant and animal cells. Two ROPinteractor proteins, PIROG1 and AtWAVE, may belong to protein complexes that are distantly related to animal WAVE complexes implicated in actin polymerization $[38,55]$. Five WAVE (or SCAR) genes might be implicated in ROP signalling [55]. All AtWAVE/SCAR genes have been found to be expressed in most organs with some exceptions and with some contradictions among various analyses $[38,71]$.

In mammalian cells, RAC is a regulator of the NADPH oxidase complex, a specialized enzyme of phagocytic cells that generates oxygen radicals to kill internalized microorganisms [4]. A cytoplasm-derived component of the plasma membrane-bound oxidase complex, p67phox, directly binds to RAC1 and RAC2 [72]. In plants, several experimental observations indicate a similar involvement of ROP GTPases into the oxidative burst-caused cell death as a pathogen- defence function [73]. The link between ROP GTPasedependent signalling and NADPH oxidase-dependent ROS generation has also been demonstrated during root development $[33,74]$. The direct interaction of rice (Oryza sativa L.) ROP GTPases and the conserved N-terminal region of OsRBOHA-D proteins, the Arabidopsis homologues of the gp91phox catalytic subunit of the NADPH-oxidase complex, has only recently been proved [58], but the family of these proteins is larger compromising 10 members. All $O s R B O H$ genes, with the exception of $O S R B O H D$ and $O s R B O H H$, are constitutively expressed in roots, shoots, leaves and calli [58].

\section{Correlative Expression of ROP Signalling-Related Genes}

Since the above protein families have many members which are not well characterized, it is very difficult to predict functional relationships among them. One possible way to clarify the picture is to investigate and compare gene expression patterns in order to restrict the predictions to those members which may occur within the same cells/tissues/ organs.

To examine the potential relationships between ROP GTPases, their regulators and effectors, a hierarchical clustering analysis of microarray expression data was performed using the Genvestigator database and analysis tools $[75,76]$. Tables 1 and 2 show the proteins/genes used for the comparison highlighting also the possibility for protein-protein interaction indicated by in vitro and/or in vivo experiments. Fig. (2) shows that many of the investigated genes are expressed in several organs/tissues, making it difficult to predict functional interactions among them. However, several proteins of these families show a preferential expression in only a small subset of organs/tissues and the correlation of the relative expression pattern of several putative partners is evident.

On Fig. (2) it is shown that five main expression clusters (A-E) could be observed. These clusters include genes with preferential or more abundant expression in the pollen and flower (clusterA), stem, node and hypocotyl (clusterB), root (clusterC), shoot and root tip, flower and silique (clusterD), and all over the plant (clusterE). Cluster E contains the highest number of genes with relatively ubiquitous expression, although preferential expression in some organs/tissues can be observed for individual genes even within this family.

The most obvious is the strong, and more or less specific, expression of a subset of genes in the pollen, including genes for ROP1,3,5 (with ROP3 and 5 expressed in many other 
tissues as well); GEF8,9,11,12,13; GDI1,2A,2B (with GDI1 also expressed at a relatively high level in vegetative tissues, especially in the root); RIC1,6; RLCK_A3,6,7; RBOHH,J. Pollen-specific expression ROP1,3,5 GTpases is well established [19] and the specific expression of a subclass of ROPGEFs in the pollen has also been confirmed by RT-PCR analysis [48]. Abundance of these transcripts in the pollen is mainly related to the establishment of polarity during pollen tube growth [46, 77, 78]. The representation of the various families with several members indicates the significance of the process the success of which is ensured by parallel pathways.

The root-enhanced expression of several other members is also clear and well defined. Interestingly, many NADPH complex subunits (RBOHA,B,C,E,F,G,I) belong to this cluster. The NADPH oxidase system has been implicated in root growth as the AtRBOHC-deficient rhd 2 mutants of Arabidospsis have short root hairs and stunted roots as consequences of defective ROS production, $\mathrm{Ca}^{2+}$ uptake and consequently cell expansion [79]. ROS formation in the root hairs is dependent on AtROP2, and this is impaired in the rhd 2 mutant, indicating a functional relationship between AtROP2 and AtRBOHC [70]. Although ROP2 [80], ROP4 and 6 [81] GTPases have been shown to play role in root hair growth, they exhibit a more ubiquitous expression pattern (Fig. 2). Their root specific interacting partners are not known, but PIROGI, SPIKE1, GAP2 and WAVE1 have the most similar expression pattern while RIC10, GAP6, GEF3,4 and 10 might be suggested as further candidates based on their preferential expression in the root hair zone (Fig. 2). RLCK_VIA5, RIC4, ROP7, GAP5, RIC2, are also expressed in the root hairs but they form a specific group due to their high relative expression in stems, nodes and hypocotyls as well (Fig. 2). WAVE3, CCR1 and GAP4 are also expressed at a relatively high level in these organs although belong to group E with a more ubiquitous expression pattern. ROP9, GEF5,6,7 and GAP1 exhibit relatively high expression in root and shoot tips in addition to other tissues. Therefore they may be important for meristem formation and maintenance.

ICR1 has also been shown to affect root meristem formation [60], although it shows stronger accumulation in the flower (petal, sepal) and the shoot apex (Fig. 2).

As it can be seen on Tables 1, 2 and Fig. (2), only few potential interactions indicated by the characteristic gene expression clusters has already been experimentally validated such as the interaction of the preferentially pollen expressed AtROP1 with RIC1,4 and GEF9 as well as NtRAC5 (ROP5) with GDI2a, in Arabidopsis and tobacco, respectively. In contrast, the functional interaction of ROPs and ROP partners with distinct expression patterns could also be demonstrated for example in epidermal cells (AtROP2,4 and AtRIC1,4 [52]) where these proteins are expressed only at a low level in comparison to other organs (Fig. 2).

\section{CONCLUSIONS}

RHO/ROP GTPase-dependent signalling pathways are complex, overlapping, interlinked and are built up by signalling modules including ROP GTPases themselves, the regulators of their GTPase cycle and downstream effectors, all coded by gene families. Signalling specificity is exerted at various levels including posttranslational modifications affecting intracellular localization and biochemical activity as well as the regulation of gene expression. In vitro as well as in vivo protein-protein interaction analysis often insufficient to reveal the biological specificity and relevance of the interaction as one protein might potentially interact with a high number of upstream regulators as well as downstream targets. Gene expression analysis may serve as a complementary approach to indicate the biological specificity and functional potential of the predicted interactions.

Obviously, the relative gene expression patterns do not allow the unambiguous prediction of potential interactions. However, there are several studies which indicate the coevolution of interacting proteins and members of signalling networks preserving similar expression pattern of the involved genes in order to ensure the presence of the partners in proper ratios [82-85]. Clustering based on gene expression may help to establish unique sets of signalling partners associated with cell types and/or signalling pathways. However, to have a clear picture a better resolution of gene expression/protein accumulation data would be required.

\section{ACKNOWLEDGEMENTS}

The author's acknowledge the support of the grants OTKA T49149 and K68064 from the Hungarian Scientific Research Fund. A.F. is also thankful for the support of the "János Bólyai" Research Fellowship.

\section{REFERENCES}

[1] Macara I, Lounsbury K, Richards S, McKiernan C, Bar-Sagi D. The Ras superfamily of GTPases. FASEB J 1996; 10: 625-30.

[2] Wennerberg K, Rossman K, Der C. The Ras superfamily at a glance. J Cell Sci 2005; 118: 843-6.

[3] Etienne-Manneville S, Hall A. Rho GTPases in cell biology. Nature 2002; 420: 629-35.

[4] Bokoch G. Regulation of innate immunity by Rho GTPases. Trends Cell Biol 2005; 15: 163-71.

[5] Brembu T, Winge P, Bones A, Yang Z. A RHOse by any other name: a comparative analysis of animal and plant Rho GTPases. Cell Res 2006; 16: 435-45.

[6] Berken A, Wittinghofer A. Structure and function of Rho-type molecular switches in plants. Plant Physiol Biochem 2008; 46(3): 380-93.

[7] Nibau C, Wu H, Cheung A. Rac/Rop GTPases: 'hubs' for signal integration and diversification in plants. Trends Plant Sci 2006; 11 : 309-15.

[8] Berken A. ROPs in the spotlight of plant signal transduction. Cell Mol Life Sci 2006; 63: 2446-59.

[9] Geyer M, Wittinghofer A. GEFs, GAPs, GDIs and effectors: taking a closer (3D) look at the regulation of Ras-related GTP-binding proteins. Curr Opin Struct Biol 1997; 7: 786-92.

[10] Sorek N, Poraty L, Sternberg H, Bar E, Lewinsohn E, Yalovsky S. Activation status-coupled transient $\mathrm{S}$ acylation determines membrane partitioning of a plant Rho-related GTPase. Mol Cell Biol 2007; 27: 2144-54.

[11] Seabra M. Membrane association and targeting of prenylated Raslike GTPases. Cell Signal 1998; 10: 167-72.

[12] Schiller M. Coupling receptor tyrosine kinases to Rho GTPases-GEFs what's the link. Cell Signal 2006; 18: 1834-43.

[13] Zheng Z, Yang Z. The Rop GTPase: an emerging signaling switch in plants. Plant Mol Biol 2000; 44: 1-9.

[14] Vernoud V, Horton A, Yang Z, Nielsen E. Analysis of the small GTPase gene superfamily of Arabidopsis. Plant Physiol 2003; 131: 1191-208.

[15] Winge P, Brembu T, Bones A. Cloning and characterization of Rac-like cDNAs from Arabidopsis thaliana. Plant Mol Biol 1997; 35: 483-95. 
[16] Moshkov I, Mur L, Novikova G, Smith A, Hall M. Ethylene regulates monomeric GTP-binding protein gene expression and activity in Arabidopsis. Plant Physiol 2003; 131: 1705-17.

[17] Brembu T, Winge P, Bones A. The small GTPase AtRac2/Rop7 is specifically expressed during late stages of xylem differentiation in Arabidopsis. J Exp Bot 2005; 56: 2465-76.

[18] Ko J, Beers E, Han K. Global comparative transcriptome analysis identifies gene network regulating secondary xylem development in Arabidopsis thaliana. Mol Genet Genomics 2006; 276: 517-31.

[19] Li H, Wu G, Ware D, Davis K, Yang Z. Arabidopsis Rho-related GTPases: differential gene expression in pollen and polar localization in fission yeast. Plant Physiol 1998; 118: 407-17.

[20] Lemichez E, Wu Y, Sanchez J, Mettouchi A, Mathur J, Chua N. Inactivation of AtRac1 by abscisic acid is essential for stomatal closure. Genes Dev 2001; 15: 1808-16.

[21] Borg S, Pødenphant L, Jensen T, Poulsen C. Plant cell growth and differentiation may involve GAP regulation of Rac activity. FEBS Lett 1999; 453: 341-5.

[22] Szücs A, Dorjgotov D, Ötvös K, et al. Characterization of three Rop GTPase genes of alfalfa (Medicago sativa L.). Biochim Biophys Acta 2006; 1759: 108-15.

[23] Kim H, Triplett B. Characterization of GhRac1 GTPase expressed in developing cotton (Gossypium hirsutum L.) fibers. Biochim Biophys Acta 2004; 1679: 214-21.

[24] Nakanomyo I, Kost B, Chua N, Fukuda H. Preferential and asymmetrical accumulation of a Rac small GTPase mRNA in differentiating xylem cells of Zinnia elegans. Plant Cell Physiol 2002; 43: 1484-92.

[25] Chan J, Peter Pauls K. Brassica napus Rop GTPases and their expression in microspore cultures. Planta 2007; 225: 469-84.

[26] Abbal P, Pradal M, Sauvage F, et al. Molecular characterization and expression analysis of the Rop GTPase family in Vitis vinifera. J Exp Bot 2007; 58(10): 2641-52.

[27] Christensen T, Vejlupkova Z, Sharma Y, et al. Conserved subgroups and developmental regulation in the monocot Rop gene family. Plant Physiol 2003; 133: 1791-808.

[28] Yamaguchi T, Hayashi T, Nakayama K, Koike S. Expression analysis of genes for callose synthases and Rho-type small GTPbinding proteins that are related to callose synthesis in rice anther. Biosci Biotechnol Biochem 2006; 70: 639-45.

[29] Luo M, Gu S, Zhao S, Zhang F, Wu N. Rice GTPase OsRacB: potential accessory factor in plant salt-stress signaling. Acta Biochim Biophys Sin (Shanghai) 2006; 38: 393-402.

[30] Yang Z, Fu Y. Rop/Rac GTPase signaling. Curr Opin Plant Biol 2007; 10(5): 490-4.

[31] Wu G, Li H, Yang Z. Arabidopsis RopGAPs are a novel family of Rho GTPase-activating proteins that require the $\mathrm{Cdc} 42 / \mathrm{Rac}-$ interactive binding motif for Rop-specific GTPase stimulation. Plant Physiol 2000; 124: 1625-36.

[32] Baxter-Burrell A, Yang Z, Springer P, Bailey-Serres J. RopGAP4dependent Rop GTPase rheostat control of Arabidopsis oxygen deprivation tolerance. Science 2002; 296: 2026-8.

[33] Carol R, Takeda S, Linstead P, et al. A RhoGDP dissociation inhibitor spatially regulates growth in root hair cells. Nature 2005; 438: $1013-6$

[34] Klahre U, Becker C, Schmitt A, Kost B. Nt-RhoGDI2 regulates Rac/Rop signaling and polar cell growth in tobacco pollen tubes. Plant J 2006; 46: 1018-31.

[35] Berken A, Thomas C, Wittinghofer A. A new family of RhoGEFs activates the Rop molecular switch in plants. Nature 2005; 436: 1176-80.

[36] Gu Y, Li S, Lord E, Yang Z. Members of a novel class of Arabidopsis Rho guanine nucleotide exchange factors control Rho GTPase-dependent polar growth. Plant Cell 2006; 18: 366-81.

[37] Thomas C, Fricke I, Scrima A, Berken A, Wittinghofer A. Structural evidence for a common intermediate in small $\mathrm{G}$ protein-GEF reactions. Mol Cell 2007; 25: 141-9.

[38] Uhrig JF, Mutondo M, Zimmermann I, et al. The role of Arabidopsis SCAR genes in ARP2-ARP3-dependent cell morphogenesis. Development 2007; 134(5): 967-77.

[39] Basu D, Le J, Zakharova T, Mallery E, Szymanski D. A SPIKE1 signaling complex controls actin-dependent cell morphogenesis through the heteromeric WAVE and ARP2/3 complexes. Proc Natl Acad Sci USA 2008; 105: 4044-9.

[40] Bischoff F, Vahlkamp L, Molendijk A, Palme K. Localization of AtRop4 and AtRop6 and interaction with the guanine nucleotide dissociation inhibitor AtRhoGDI1 from Arabidopsis. Plant Mol Biol 2000; 42: 515-30.

[41] Michaelson D, Silletti J, Murphy G, D'Eustachio P, Rush M, Philips M. Differential localization of Rho GTPases in live cells: regulation by hypervariable regions and RhoGDI binding. J Cell Biol 2001; 152: 111-26.

[42] Tao L, Cheung A, Wu H. Plant Rac-like GTPases are activated by auxin and mediate auxin-responsive gene expression. Plant Cell 2002; 14: 2745-60.

[43] Kieffer F, Elmayan T, Rubier S, Simon-Plas F, Dagher MC, Blein JP. Cloning of Rac and Rho-GDI from tobacco using an heterologous two-hybrid screen. Biochimie 2000; 82: 1099-105.

[44] Peck J, Douglas G4, Wu C, Burbelo P. Human RhoGAP domaincontaining proteins: structure, function and evolutionary relationships. FEBS Lett 2002; 528: 27-34.

[45] Klahre U, Kost B. Tobacco RhoGTPase ACTIVATING PROTEIN1 spatially restricts signaling of RAC/Rop to the apex of pollen tubes. Plant Cell 2006; 18: 3033-46.

[46] Kost B. Spatial control of Rho (Rac-Rop) signaling in tip-growing plant cells. Trends Cell Biol 2008; 18: 119-27.

[47] Shichrur K, Yalovsky S. Turning on the switch--RhoGEFs in plants. Trends Plant Sci 2006; 11: 57-9.

[48] Zhang Y, McCormick S. A distinct mechanism regulating a pollenspecific guanine nucleotide exchange factor for the small GTPase Rop in Arabidopsis thaliana. Proc Natl Acad Sci USA 2007; 104: 18830-5.

[49] Qiu J, Jilk R, Marks M, Szymanski D. The Arabidopsis SPIKE1 gene is required for normal cell shape control and tissue development. Plant Cell 2002; 14: 101-18.

[50] Côté J, Vuori K. GEF what? Dock180 and related proteins help Rac to polarize cells in new ways. Trends Cell Biol 2007; 17: 38393.

[51] Cotteret S, Chernoff J. The evolutionary history of effectors downstream of Cdc42 and Rac. Genome Biol 2002; 3: REVIEWS0002.

[52] Fu Y, Gu Y, Zheng Z, Wasteneys G, Yang Z. Arabidopsis interdigitating cell growth requires two antagonistic pathways with opposing action on cell morphogenesis. Cell 2005; 120: 687-700.

[53] Wu G, Gu Y, Li S, Yang Z. A genome-wide analysis of Arabidopsis Rop-interactive CRIB motif-containing proteins that act as Rop GTPase targets. Plant Cell 2001; 13: 2841-56.

[54] Gu Y, Fu Y, Dowd P, et al. A Rho family GTPase controls actin dynamics and tip growth via two counteracting downstream pathways in pollen tubes. J Cell Biol 2005; 169: 127-38.

[55] Basu D, El-Assal S, Le J, Mallery E, Szymanski D. Interchangeable functions of Arabidopsis PIROGI and the human WAVE complex subunit SRA1 during leaf epidermal development. Development 2004; 131: 4345-55.

[56] Kawasaki T, Koita H, Nakatsubo T, et al. Cinnamoyl-CoA reductase, a key enzyme in lignin biosynthesis, is an effector of small GTPase Rac in defense signaling in rice. Proc Natl Acad Sci USA 2006; 103: 230-5.

[57] Hong Z, Zhang Z, Olson J, Verma D. A novel UDP-glucose transferase is part of the callose synthase complex and interacts with phragmoplastin at the forming cell plate. Plant Cell 2001; 13: 76979.

[58] Wong HL, Pinontoan R, Hayashi K, et al. Regulation of rice NADPH oxidase by binding of Rac GTPase to its N-terminal extension. Plant Cell 2007; 19: 4022-34.

[59] Molendijk A, Ruperti B, Singh M, et al. A cysteine-rich receptorlike kinase NCRK and a pathogen-induced protein kinase RBK1 are Rop GTPase interactors. Plant J 2008; 53: 909-23.

[60] Lavy M, Bloch D, Hazak O, et al. A novel Rop/Rac effector links cell polarity, root-meristem maintenance, and vesicle trafficking. Curr Biol 2007; 17: 947-52.

[61] Thao N, Chen L, Nakashima A, et al. RAR1 and HSP90 form a complex with Rac/Rop GTPase and function in innate-immune responses in rice. Plant Cell 2007; 19: 4035-45.

[62] Zhao Z, Manser E. PAK and other Rho-associated kinases-effectors with surprisingly diverse mechanisms of regulation. Biochem J 2005; 386: 201-14.

[63] Bokoch G. Biology of the p21-activated kinases. Annu Rev Biochem 2003; 72: 743-81.

[64] Dan I, Watanabe N, Kusumi A. The Ste20 group kinases as regulators of map kinase cascades. Trends Cell Biol 2001; 11: 220-30.

[65] Lieberherr D, Thao NP, Nakashima A, Umemura K, Kawasaki T, Shimamoto K. A sphingolipid elicitor-inducible mitogen-activated 
protein kinase is regulated by the small GTPase OsRac1 and heterotrimeric G-protein in rice. Plant Physiol 2005; 138: 1644-52.

[66] Jurca M, Bottka S, Fehér A. Characterization of a family of Arabidopsis receptor-like cytoplasmic kinases (RLCK class VI). Plant Cell Rep 2008; 27: 739-48.

[67] Kost B, Lemichez E, Spielhofer P, et al. Rac homologues and compartmentalized phosphatidylinositol 4, 5-bisphosphate act in a common pathway to regulate polar pollen tube growth. J Cell Biol 1999; 145: 317-30.

[68] Oude Weernink PA, Schmidt M, Jakobs KH. Regulation and cellular roles of phosphoinositide 5-kinases. Eur J Pharmacol 2004; 500: 87-99.

[69] Hwang J, Gu Y, Lee Y, Yang Z. Oscillatory Rop GTPase activation leads the oscillatory polarized growth of pollen tubes. Mol Biol Cell 2005; 16: 5385-99.

[70] Fu Y, Wu G, Yang Z. Rop GTPase-dependent dynamics of tiplocalized F-actin controls tip growth in pollen tubes. J Cell Biol 2001; 152: 1019-32.

[71] Frank M, Egile C, Dyachok J, et al. Activation of ARP2/3 complex-dependent actin polymerization by plant proteins distantly related to SCAR/WAVE. Proc Natl Acad Sci USA 2004; 101: 16379-84.

[72] Dorseuil O, Reibel L, Bokoch G, Camonis J, Gacon G. The Rac target NADPH oxidase p67phox interacts preferentially with Rac2 rather than Rac1. J Biol Chem 1996; 271: 83-8.

[73] Agrawal G, Iwahashi H, Rakwal R. Small GTPase 'Rop': molecular switch for plant defense responses. FEBS Lett 2003; 546: 173-80.

[74] Jones M, Raymond M, Yang Z, Smirnoff N. NADPH oxidasedependent reactive oxygen species formation required for root hair growth depends on Rop GTPase. J Exp Bot 2007; 58: 1261-70.

[75] Grennan A. Genevestigator. facilitating web-based gene-expression analysis. Plant Physiol 2006; 141: 1164-6.
[76] Zimmermann P, Hirsch-Hoffmann M, Hennig L, Gruissem W. Genevestigator. Arabidopsis microarray database and analysis toolbox. Plant Physiol 2004; 136: 2621-32.

[77] Fu Y, Yang Z. Rop GTPase: a master switch of cell polarity development in plants. Trends Plant Sci 2001; 6: 545-7.

[78] Cole R, Fowler J. Polarized growth: maintaining focus on the tip. Curr Opin Plant Biol 2006; 9: 579-88.

[79] Foreman J, Demidchik V, Bothwell J, et al. Reactive oxygen species produced by NADPH oxidase regulate plant cell growth. Nature 2003; 422: 442-6.

[80] Jones M, Shen J, Fu Y, Li H, Yang Z, Grierson C. The Arabidopsis Rop2 GTPase is a positive regulator of both root hair initiation and tip growth. Plant Cell 2002; 14: 763-76.

[81] Molendijk A, Bischoff F, Rajendrakumar C, et al. Arabidopsis thaliana Rop GTPases are localized to tips of root hairs and control polar growth. EMBO J 2001; 20: 2779-88.

[82] Ettwiller L, Veitia RA. Protein coevolution and isoexpression in yeast macromolecular complexes. Comp Funct Genomics 2007; 58721.

[83] Casneuf T, De Bodt S, Raes J, Maere S, Van de Peer Y. Nonrandom divergence of gene expression following gene and genome duplications in the flowering plant Arabidopsis thaliana. Genome Biol 2006; 7: R13.

[84] Duarte JM, Cui L, Wall PK, et al. Expression pattern shifts following duplication indicative of subfunctionalization and neofunctionalization in regulatory genes of Arabidopsis. Mol Biol Evol 2006; 23: 469-78.

[85] Fraser HB, Hirsh AE, Wall DP, Eisen MB. Coevolution of gene expression among interacting proteins. Proc Natl Acad Sci USA 2004; 101: 9033-8.

(C) Fehér et al.; Licensee Bentham Open.

This is an open access article licensed under the terms of the Creative Commons Attribution Non-Commercial License (http://creativecommons.org/licenses/by-nc/3.0/) which permits unrestricted, non-commercial use, distribution and reproduction in any medium, provided the work is properly cited. 